J. Klin. Endokrinol. Stoffw. 2019 · 12:159-164 https://doi.org/10.1007/s41969-019-0071-x Online publiziert: 12. August 2019

(c) Der/die Autor(en) 2019

\author{
Michael Weissel ${ }^{1,2}$ \\ 'Wien, Österreich \\ ${ }^{2}$ Medical University of Vienna, Wien, Österreich
}

\title{
Rolle der bioidenten Schilddrüsenhormone in der Behandlung der Hypothyreose: Ein Update
}

Seit der Erkenntnis von Theodor Kocher [1] und Victor Horsley [2], dass die Schilddrüse kein nutzloses Organ ist, wird versucht, die fehlenden Hormone nach Kropfexstirpation bzw. bei Unterfunktion der Schilddrüse zu ersetzen.

Ende des 19. Jahrhunderts standen drei Möglichkeiten der Ersatztherapie zur Verfügung:

1. Die Transplantation tierischer [3] oder auch menschlicher Schilddrüsen, wobei das menschliche Gewebe von Patienten entnommen wurde, die wegen Knoten operiert wurden. Der Ort der Transplantation wurde vor allem nach der Durchblutung ausgesucht [4]. Unterstützt wurde diese Therapie durch die Gabe von Extrakten tierischer Schilddrüsen.

2. Flüssiger Schilddrüsenextrakt: Die Therapie eines Myxödems mit subkutan verabreichtem flüssigem Extrakt einer Schilddrüse vom Schaf wurde erstmals 1890 von Bettencourt und Serrano [5] und kurz danach von Murray [6] beschrieben. Murray konnte 25 Jahre später bei derselben Patientin auch den Langzeiterfolg einer lebenslangen subkutanen Therapie berichten [7].

3. Getrockneter Schilddrüsenextrakt: 1892 haben Fox [8], Mac Kenzie [9] und Vermeulen [10] unabhängig voneinander zeigen können, dass oral verabreichter getrockneter Extrakt von tierischen Schilddrüsen die Symptome der Hypothyreose ebenfalls deutlich bessert. Der Schilddrüsenextrakt war „gereinigte, getrocknete und pulverisierte tierische (Schaf, Rind, Schwein) Schilddrüse nach Entfernung des Fetts und des Bindegewebes".

Die Behandlung der Hypothyreose mit getrockneten Schilddrüsenextrakten kam aber bald in das Kreuzfeuer der Kritik, da es Probleme bezüglich der Verlässlichkeit des Hormongehalts der Schilddrüsenextrakte gab und da es wegen des manchmal relativ hohen Trijodthyronin(T3)-Anteils zu einem Anstieg dieses Hormons (auch in den hyperthyreoten Bereich) kam. Eine klinisch wirksame Hyperthyreose durch solche Präparate wurde noch 1989 von Sawin und London [11] beschrieben.

Die Isolation von Thyroxin (T4) durch Kendall [12] und dessen Synthese durch Harington[13] bzw. einige Zeit später die Entwicklung des besser resorbierbaren Na-Salzes von T4 [14] stellten bereits relativ früh (ab 1949) eine gute Alternative dar. Der Nachweis der peripheren - extrathyreoidalen - Konversion des T4 in das biologisch aktivere T3 durch Braverman, Ingbar und Sterling [15] gab die theoretische Grundlage dafür, dass die Monotherapie mit T4 ausreichend ist.

Trotzdem waren noch 1976 in den USA ungefähr $52 \%$ der Verschreibungen für Schilddrüsenhormonersatz getrockneter Schilddrüsenextrakt oder andere Naturprodukte [16]. Heute hat sich das drastisch geändert: Na-L-Thyroxin ist in den USA eines der am häufigsten verschriebenen Medikamente [17].
Allerdings sind ca. 10-15\% der hypothyreoten Patienten, die unter einer T4-Monotherapie normale TSH-Spiegel aufweisen, mit ihrer Lebensqualität unzufrieden. Sie klagen über weiter bestehende Symptome einer Hypothyreose $[18,19]$ und vor allem über neurokognitive Einschränkungen [20]. Interessant ist in diesem Zusammenhang, dass ca. $15 \%$ von athyreoten Patienten unter einer L-T4-Monotherapie keine normalen T3-Spiegel erreichen [21].

Da Lebensqualität als solche ein schwierig zu erfassender Begriff ist, stellt eine Objektivierung dieser Einschränkung eine große Herausforderung dar. So versuchte man, andere Biomarker als das TSH zu testen: Samuels et al. [22] konnten zum Beispiel 2016 zeigen, dass der Grundumsatz von hypothyreoten $\mathrm{Pa}$ tienten mit normalem TSH im Vergleich $\mathrm{zu}$ einem Kontrollkollektiv signifikant verringert - aber im Normalbereich war. Eine rezente große Metaanalyse [23] von 65 Studien ergab, dass nicht alle bekannten - von der Schilddrüse abhängigen - Biomarker im Normbereich bzw. von der gesunden Kontrollgruppe verschieden waren. Das Gesamt-Cholesterin und sein LDL-Anteil waren bei hypothyreoten Patienten mit normalem TSH unter L-T4 sowohl gegen Kontrollen als auch zum Normalbereich gering erhöht.

Die erhoffte Lösung des Problems durch die gleichzeitige Verabreichung von L-T4 und L-T3 schlug aber auch fehl: Eine doppelblinde plazebokontrollierte Studie von 2000-2002 an 46 Patienten 
zeigte nach vier Monaten keine Überlegenheit einer L-T4/L-T3-Kombinationstherapie im Vergleich zur Monotherapie mit L-T4 [24]. Nach Durchsicht der wenigen seriösen Folgestudien raten sowohl die europäischen [18] als auch die amerikanischen [19] Leitlinien von einer Kombinationstherapie T4 plus T3 als prinzipielle Alternative zur T4-Monotherapie ab. Die europäischen Guidelines schwächen ihre Ablehnung durch die Empfehlung ab, dass ein L-T4/LT3Therapieversuch bei hypothyreoten $\mathrm{Pa}$ tienten, die trotz TSH im Normalbereich weiterhin Beschwerden haben, in Erwägung zu ziehen sei. Dieser Therapieversuch sollte nach drei Monaten evaluiert und bei Erfolglosigkeit abgebrochen werden. Die amerikanischen Guidelines empfehlen ganz klar, von einer Kombinationstherapie abzusehen („recommend against“).

Eine 2017 unter den Mitgliedern der American Thyroid Association durchgeführte Umfragestudie [25] zeigte, dass die Kollegen in den USA diese Guidelines eher nicht befolgen und häufiger Alternativen zur T4-Monotherapie suchen als die Ärzte aus Europa oder anderen Regionen. Einschränkend sei darauf hingewiesen, dass Umfragestudien immer das Problem der Unsicherheit haben, wie repräsentativ die Beantworter der Fragebogen für die Fragestellung sind. Außerdem spielt natürlich auch die Verfügbarkeit alternativer Möglichkeiten eine Rolle. In Österreich ist es zum Beispiel nicht einfach, an Thyreoidea siccata (DTE) heranzukommen.

Eine Ausnahme scheint es aber zu geben: Hypothyreote Patienten mit Variationen im Dejodinase-2-Gen klagen laut Panicker et al. [26] häufiger über psychische Beschwerden. Diese psychischen Missempfindungen sprachen bei den von den Autoren beobachteten Patienten signifikant besser auf eine T4/T3-Kombinationstherapie an als ebenso behandelte hypothyreote Patienten mit intakter Dejodinase $\mathrm{DIO} 2$.

Die amerikanischen Leitlinien [19] nehmen auf die sich daher ergebende Frage, ob genetische Varianten des Schilddrüsenhormonabbaus während der Substitutionstherapie hypothyreoter Patienten eine Rolle spielen, ziemlich unbeeindruckt von Panickers Studie folgendermaßen Bezug:

Spezifische Polymorphismen der Deiodinasen bewirken sehr geringe Änderungen der Serumkonzentrationen der Schilddrüsenhormone. Es gibt keine ausreichenden Daten, ob Polymorphismen der Dejodinase-oder Transportergene klinisch relevante Wirkungen aufGewebskonzentrationen der Schilddrüsenhormone ausüben.

Die europäischen Leitlinien [18] geben hierzu keine Empfehlung ab - mit dem oben besprochenen in Erwägung zu ziehenden dreimonatigen Therapieversuch bei Patienten, die sich unter adäquater T4-Monotherapie schlecht fühlen, werden diese Patienten aber ohnehin miterfasst.

Die T4-Monotherapie macht also nicht alle hypothyreoten Patienten glücklich. Die Kombinationstherapie mit ihrem handelsüblichen unphysiologischen Verhältnis von T4:T3 von 4-5:1 (physiologisch wäre 15:1) wird von den Experten abgelehnt - was bleibt also übrig?

\section{Extrakte von tierischen Schilddrüsen (,Desiccated Thyroid Extract" = DTE)}

Bis Ende der 50er Jahre war Thyranon $\mathrm{R}$ (Fa. Organon) - ein Extrakt aus Rinderschilddrüsen - in Österreich das marktbestimmende Medikament zur Substitution der Hypothyreose. Es gab Tabletten mit 50 und $100 \mathrm{mg}$, wobei der Hormongehalt nicht angegeben wurde, sondern nur, dass die Tablette zu $30 \%$ aus organisch gebundenem Jod besteht.

Die US-Pharmacopeia legt heute den Hormongehalt des tierischen (Schwein) Schilddrüsenextrakts relativ genau fest: Es handelt sich um „von Fett und Bindegewebe gereinigten, pulverisierten und getrockneten Schilddrüsenextrakt", der $38 \mu \mathrm{g}( \pm 15 \%) \quad$ L-Thyroxin und $9 \mu \mathrm{g}$ (+10\%) L-3,5,3'-Trijodthyronin pro Grain $(=60-65 \mathrm{mg})$ enthalten soll. Der zusätzliche Inhalt dieser Extrakte, wie Mono- und Dijodthyronin, Thyronamine und Calcitonin wird allerdings nicht festgelegt.

Thyreoidea siccata (DTE) ist sowohl in den USA als auch in Europa verschreibungspflichtig und nur über Apotheken erhältlich. In den USA ist es zwar nicht von der FDA approbiert, aber da es schon vor dem Inkrafttreten der heute gültigen Zulassungsbestimmungen der FDA am Markt war („grandfathered in“ -persönliche Mitteilung von Jacqueline Jonklaas 14.05.2019), benötigt es eine solche Approbation nicht. Ein entsprechendes Ansuchen um Zulassung wurde von den Firmen daher bisher nie gestellt. Die Folge der fehlenden Approbation ist, dass die FDA die Hormonzusammensetzung der Extraktpräparationen nicht regelmäßig überprüft [27].

Wie bei vielen Naturprodukten, die in Konkurrenz zu synthetisch hergestellten Produkten der Schulmedizin stehen, entwickelt sich eine sehr emotionale, oft irrationale Diskussion. Hypothyreote Patienten, die sich unter der Monotherapie nicht wohl fühlen, fordern manchmal vehement eine Verschreibung dieser Schilddrüsenextrakte ein.

Ein Preisvergleich zu den synthetischen Hormonen ist der $\bullet$ Tab. 1 zu entnehmen. Da Thyreoidea siccata in Österreich mehr als $10 \times$ so teuer ist, muss das Argument für eine Verschreibung sehr valide sein.

Die amerikanischen Leitlinien [19] empfehlen, dass die L-T4-Monotherapie der Therapie mit Thyroidea siccata vorgezogen wird („strong recommendation, moderate quality evidence"). Sie äußern nämlich Sicherheitsbedenken gegen diese Therapie: Einerseits befürchten sie hyperthyreote Phasen durch den supraphysiologischen L-T3-Gehalt, andererseits bemängeln sie das Fehlen von publizierten Langzeitsicherheitsstudien.

Es gibt bisher nur eine einzige randomisierte doppelblinde Cross-over-Studie, die die Behandlung hypothyreoter Patienten mit L-T4 mit dem Erfolg der Behandlung mit DTE vergleicht [28]: In dieser Studie von Hoang et al. wurden insgesamt 70 Patienten untersucht, wobei jeweils 35 Patienten zuerst DTE bzw. L-T4-Monotherapie 16 Wochen in einer Dosierung gegeben wurde, die normale TSH-Spiegel ergab. Nach 16 Wochen wurde die Therapie von DTE aufL-T4 beziehungsweise vice versa umgesetzt. Die Patienten wurden am Beginn der Studie und am Ende jeder Behandlungsperiode zahlreichen neurokognitiven Tests unter- 
Hier steht eine Anzeige.

\section{曾 Springer}




\section{Weissel}

\section{Rolle der bioidenten Schilddrüsenhormone in der Behandlung der Hypothyreose: Ein Update}

\section{Zusammenfassung}

Die vorliegende kurze Übersicht nimmt zur Rolle der bioidenten Schilddrüsenhormone (Desiccated Thyroid Extract $=$ DTE) bei der Behandlung der Hypothyreose Stellung. DTE steht bereits seit dem Ende des 19. Jahrhunderts zur Verfügung. Anfangs wurden die Schilddrüsen aller möglichen Haustiere (Schaf, Rind, Schwein) verwendet. Heute sind es vor allem Schweineschilddrüsen, die zum Einsatz kommen.

Bald stellte die Unverlässlichkeit des Inhalts der Extrakte ein Problem dar. Die US-Pharmacopeia legte daher den Gehalt an T4 und T3 fest. Zum restlichen Inhalt wird nicht Stellung genommen. Eine Approbation der Food and Drug Administration (FDA) liegt nicht vor - ist aber auch nicht obligatorisch, da DTE bereits vor der Erstellung der Approbationsregeln am Markt war („grandfathered in“). Die Folge davon ist, dass der Inhalt der DTE-Präparate nicht von der FDA überprüft wird. Sowohl in den USA als auch in den meisten europäischen Ländern ist DTE verschreibungspflichtig und kann nur über Apotheken erhalten werden. Die Standardtherapie der Hypothyreose ist die L-Thyroxin(T4)-Monotherapie. Ein Preisvergleich in Österreich zeigt, dass DTE zehnmal so teuer ist wie L-T4. Sowohl die europäischen als auch die amerikanischen Leitlinien stehen dem Einsatz von DTE sehr kritisch gegenüber. Die Hauptvorwürfe gegen DTE sind der hohe Anteil an L-3,5,3'Trijodthyronin (T3) und das Fehlen von Sicherheitslangzeitstudien. Die Leitlinien empfehlen beide die L-T4-Monotherapie zur Behandlung der Hypothyreose. Ein direkter Vergleich zeigte in der einzigen vorliegenden doppelblinden Cross-over-Studie keinen messbaren Vorteil der Lebensqualität unter der Therapie mit DTE. Die Mehrheit der doppelblind behandelten Patienten entschied sich allerdings nach Entblindung für eine Fortsetzung der Therapie mit DTE. Aufgrund der fehlenden Evidenz scheint nur bei jenen - wenigen - hypothyreoten Patienten, die unter einer adäquaten (TSH monatelang im Normbereich) L-T4-Monotherapie noch immer Beschwerden haben, ein Therapieversuch mit DTE gerechtfertigt. Dieser sollte nach drei Monaten evaluiert werden. Abschließend wird noch die sehr unterschiedliche Akzeptanz der Leitlinien durch die behandelnden Ärzte und ihre Patienten diskutiert.

\section{Schlüsselwörter}

Hypothyreose $\cdot$ L-Thyroxin $\cdot$ Thyreoidea siccata $\cdot$ Monotherapie $\cdot$ Kombinationstherapie

\section{Role of desiccated thyroid extracts in the treatment of hypothyroidism: an update}

\section{Abstract}

This short overview comments on the role of bioactive thyroid hormones (desiccated thyroid extract [DTE]) in the treatment of hypothyroidism. DTE has been available since the end of the 19th century. Cattle, sheep and pig thyroids used to be the main sources. Today, mainly pig thyroid glands are used. However, the varying content of thyroid hormones in these extracts has become a problem. Therefore, the US Pharmacopeia defined the content of L-thyroxine (T4) and L-3,5, '3'-triiodothyronine (T3), but without defining the content of the other compounds present in animal thyroids.

DTE is not approved by the Food and Drug Administration (FDA). Approval does not seem to be necessary since it was "grandfathered in." (Substances on the market before new FDA approval rules were implemented do not need approval.) The consequence of this fact is that the FDA does not examine the content and quality of DTE. In the US and in most European countries DTE can only be purchased in pharmacies with a prescription. L-Thyroxine monotherapy is the standard treatment for hypothyroidism. A price comparison in Austria shows that DTE is at least ten times more expensive than L-T4. European and even more so US guidelines recommend against DTE. The US guidelines argue that the relatively high $\mathrm{T} 3$ proportion (T4:T3 being 4:1) bears the danger of hyperthyroidism and that longtime safety studies are lacking. There is only one shortterm study that compares L-T4 with DTE treatment of hypothyroidism. This doubleblind cross over study shows no evidence of an advantage of DTE. The parameters of quality of life were not significantly different. However, the majority of the unblinded patients would have preferred continuation of treatment with DTE.

European guidelines suggest trying DTE (or T4/T3 combination therapy) only in hypothyroid patients who continue to complain of symptoms in spite of adequate L-T4 monotherapy for several months. DTE treatment should be re-evaluated after three months and stopped if there is no evidence of success. Finally, this short review also discusses the acceptance of the guidelines by the treating physicians and their patients.

Keywords

Hypothyroidism · L-Thyroxine · Desiccated Thyroid Extract · Monotherapy . Combinationtherapy zogen - zusätzlich wurden biochemische Biomarker der Schilddrüsenfunktion getestet. Das Ergebnis der Studie wird folgendermaßen von den Autoren zusammengefasst:

Die DTE-Therapie führte zu keiner signifikanten Besserung der Lebensqualität. Allerdings äußerten fast die Hälfte (49\%) der Patienten retrospektiv eine Präferenz der Therapie mit DTE im Vergleich zur Behandlung mit L-T4 (19\%). Die Therapie mit DTE mag für manche hypothyreote Patienten von Relevanz sein.

Objektiv gibt es also bisher keine Evidenz für einen Vorteil der Therapie mit DTE - subjektiv scheinen sich jedoch man- che Patienten undefinierbar unter DTE besser $\mathrm{zu}$ fühlen.

Die 2014 publizierten Leitlinien der American Thyroid Association (ATA) [19] weisen außerdem auf das Fehlen von Langzeitstudien einer Therapie der Hypothyreose mit DTE hin. 2018 ist eine qualitativ anfechtbare Studie $\mathrm{zu}$ dem Thema erschienen [29]: es handelt 


\begin{tabular}{|c|c|c|c|}
\hline & $\begin{array}{l}\text { Thyreoidea siccata } \\
\text { (Armour Thyroid) }\end{array}$ & $\begin{array}{l}\text { L-Thyroxin } \\
\text { (Euthyrox) }\end{array}$ & $\begin{array}{l}\text { L-Thyroxin + L-T3 } \\
\text { (Novothyral) }\end{array}$ \\
\hline $\begin{array}{l}\text { Inhalt pro Tabl. } \\
\text { (grain) }\end{array}$ & $\begin{array}{l}38 \mu \mathrm{g} \mathrm{L}-\mathrm{T} 4+ \\
9 \mu \mathrm{g} \mathrm{L}-\mathrm{T3}\end{array}$ & $100 \mu \mathrm{g}$ & $\begin{array}{l}100 \mu \mathrm{g} \mathrm{L-T4}+ \\
20 \mu \mathrm{g} \text { L-T3 }\end{array}$ \\
\hline Preis/100 Stück (€) & 112,4 & 8,60 & 10,35 \\
\hline Info Quelle & $\begin{array}{l}\text { Weltapotheke in Wien } \\
(19.01 .2019)\end{array}$ & Austria Codex 2019 & Austria Codex 2019 \\
\hline
\end{tabular}

sich dabei um eine retrospektive, nicht randomisierte Beobachtungsstudie des Zeitraums 2010-2016. Hundert hypothyreote Patienten, die unter einer L-T4Monotherapie trotz normaler TSH-Spiegel weiter symptomatisch waren, wurden untersucht. Bei niedrig-normalem freiem T3 wurden die Patienten entweder auf DTE $(n=57 ; 30 \mathrm{mg} / \mathrm{Tag})$ oder auf eine Therapie mit einer T4/T3-Kombination im Verhältnis 15:1 $\quad(N=37$; mittlere Dosis $75 \mu \mathrm{g}$ T4/5 $\mu$ g T3/Tag) umgestellt. Die mittlere Beobachtungsdauer war 27 Monate (1-111 Monate). Unverständlich ist, wie mit der niedrigen Dosis von DTE $(30 \mathrm{mg}=$ ca. $1 / 2$ grain $=20 \mu \mathrm{g}$ T4 und $5 \mu \mathrm{g}$ T3) dieselben normalen TSH-Spiegel erreicht werden konnten wie mit der vergleichsweise viel höheren Dosis der Kombinationstherapie. Soweit der etwas verwirrenden Darstellung der Ergebnisse zu entnehmen ist, wurde kein erhöhtes Risiko für das Auftreten von Hospitalisierungen wegen Vorhofflimmern, kardiovaskulären Erkrankungen oder Mortalität bei Patienten aller Altersklassen beobachtet.

In letzter Zeit sind zwei Umfragestudien zu dem Thema erschienen, bei denen entweder an Ärzte oder an Patienten Fragebögen ausgeschickt wurden: Shresta et al. [30] haben eine Umfrage über mögliche Nebenwirkungen einer Therapie mit DTE unter den Mitgliedern der American Thyroid Association, der Endocrine Society und der American Association of Clinical Endocrinologists durchgeführt. 174 Kollegen berichteten über Nebenwirkungen, wobei $68 \%$ der Patienten Symptome oder/und TSHWerte wie bei Hyperthyreose hatten. Zwei Patienten hatten eine dokumentierte neu aufgetretene Arrhythmie.
Die andere - viel größere - Studie [31] befragte hypothyreote Patienten, an die sie über die Mitglieder der American Thyroid Association herankam, mittels über das Internet ausgesandter umfangreicher Fragebögen nach ihrer Lebensqualität sowie abschließend auch nach der Zufriedenheit mit ihren betreuenden Ärzten. Die Patienten standen entweder unter einer Monotherapie mit L-T4 $(n=6949)$, oder einer T4/T3-Kombinationstherapie $(n=978)$ oder einer Therapie mit DTE $(n=3239)$. Die Auswertung der Fragebögen erfolgte mit einer Skala von 1 (sehr unzufrieden) bis 10 (sehr zufrieden), die den Grad der Zufriedenheit wiedergab: Die L-T4-Monotherapie schnitt am schlechtesten mit der Note 5 (Interquartilbereich: 3-7), die DTE-Therapie am besten mit der Note 7 (Interquartilbereich 5-9) im Mittel ab. Insgesamt waren die hypothyreoten Patienten unter DTE mit ihrem Zustand und interessanterweise auch mit ihren Ärzten zufriedener als die Vergleichsgruppen. Wahrscheinlich waren sie auch wesentlich motivierter, die Fragebögen auszufüllen.

Zusammenfassend muss also festgestellt werden, dass es 1) keine klare Evidenz dafür gibt, dass die Lebensqualität hypothyreoter Patienten unter DTE besser ist als unter der Monotherapie mit L-Thyroxin. Allerdings scheint der subjektive Zufriedenheitsgrad unter DTE undefinierbar besser zu sein.

2) bis heute keine soliden Studien darüber vorliegen, ob eine Langzeittherapie mit DTE mit relevanten Nebenwirkungen behaftet ist (Hyperthyreose durch T3-Anteil?). Da es sich um eine lebenslange Therapie handelt, müssen solche Studien gefordert werden.

3) Der Preisvergleich - zumindest in Österreich - ein zusätzliches Argument dafür ist, die DTE-Therapie wirklich nur als Therapieversuch nach fehlendem Therapieerfolg einer adäquaten T4-Monotherapie einzusetzen.

\section{Korrespondenzadresse

Univ. Prof. Dr.
Michael Weissel
Schlösselgasse 1/3,
1080 Wien, Österreich
michael.weissel@
meduniwien.ac.at

Funding. Open access funding provided by Medical University of Vienna.

\section{Einhaltung ethischer Richtlinien}

Interessenkonflikt. M. Weissel gibt an, dass kein Interessenkonflikt besteht.

Für diesen Beitrag wurden von den Autoren keine Studien an Menschen oder Tieren durchgeführt. Für die aufgeführten Studien gelten die jeweils dort angegebenen ethischen Richtlinien.

Open Access. Dieser Artikel wird unter der Creative Commons Namensnennung 4.0 International Lizenz (http://creativecommons.org/licenses/by/4.0/deed. de) veröffentlicht, welche die Nutzung, Vervielfältigung, Bearbeitung, Verbreitung und Wiedergabe in jeglichem Medium und Format erlaubt, sofern Sie den/die ursprünglichen Autor(en) und die Quelle ordnungsgemäßnennen, einen Link zur Creative Commons Lizenz beifügen und angeben, ob Änderungen vorgenommen wurden.

\section{Literatur}

1. Kocher $T$ (1883) Über Kropfexstirpation und ihre Folgen. Arch Klin Chir 39(1883):254-335

2. Horsley V (1884) Remarks on the function of the thyroid gland. Proc Royal Soc Lond (1854-1905) 38:5-7

3. Eiselsberg A (1892) Über erfolgreiche Einheilung der Katzenschilddrüse in die Bauchdecke und Auftreten von Tetanie nach deren Exstirpation. Wien Klin Wochenschr 5:81-85

4. Kocher A (1923) The treatment of hypothyroidism by transplantation. BrMed J 1:560

5. Bettencourt R, Serrano J-A (1890) Un cas de myxœdème (cachexie pachydermique) traité par la greffe hypodermique du corps thyroïde d'un mouton. Compte rendu de la 19eme session de I'Association française pour l'avancement des sciences. , Limoges, S683-690

6. Murray GR (1891) Note on the treatment of myxedema by hypodermic injections of an extract of the thyroid gland of a sheep. BrMed J 1891:2796

7. Murray GR (1920) The life history of the first case of Myxedema treated by thyroid extract. Br Med J 1920:359

8. Fox EL (1892) A case of Myxedema treated by taking extract of thyroid by mouth. BrMed J 2:941 
9. MacKenzie HWG (1892) A case of Myxedema treated with great benefit by feeding with fresh thyroid glands. Br Med J 2:940

10. Vermeulen $F$ (1893) The treatment of Myxedema by feeding with thyroid glands. Br Med J 1:266

11. Sawin CT, London MH (1989) Natural desiccated thyroid. A "health food" thyroid preparation. Arch Intern Med 149:2117-2118

12. Kendall EC (1915) The isolation in crystalline form of the compound containing iodine which occurs in the thyroid. Its chemical nature and physiologic activity. JAMA 64:2042

13. Harington CR, Barger G (1927) III. Constitution and Synthesis of Thyroxine. Biochem J 21:169

14. Chalmers J, Dickinson G, Elks J, Hems BA (1949) The synthesis of Thyroxine and related substances. Part V. A synthesis of L-Thyroxine from L-Tyrosine. JChem Soc 1949:3424-3438

15. Bravermann LE, Ingbar SH, Sterling K (1970) Conversion of Thyroxine to Triiodothyronine in Athyreotic human subjects. J Clin Invest 49:855-864

16. Donahue JA, (national Prescription Audit, IMS America) (1978) Persönliche Mitteilung an CT Sawin. zitert nach Sawin CT et al. 1978: A Comparison of Thyroxine and Desiccated Thyroid in Patients with Primary Hypothyroidism. Metabolism 27:1518

17. Durham NC (2017) Medicine Use and Spending in the U.S. A Review of 2016 and Outlook to 2021. QuintilesIMS Institute. IQVIA Institute for Human DataScience,

18. Wiersinga WM, Duntas L, Fadeyev V, Nygaard B, Vanderpump MPJ (2012) 2012 ETA guidelines: the use of L-T4 + L-T3 in the treatment of hypothyroidism. Eur Thyroid J 1:55-71

19. Jonklaas J, Bianco AC, Bauer AJ, Burman KD, Cappola AR, Celi FS, Cooper DS, Kim B, Peeters R, Rosenthal MS, Sawka A (2014) Guidelines for the Treatment of Hypothyroidism: prepared by the American Thyroid Association Task Force on Thyroid Hormone Replacement. Thyroid 24:1670-1751

20. Saravanan P, Chau WF, Roberts N, Vedhara K, Greenwood R, Dayan CM (2002) Psychoological well-being in patients on "adequate" doses of L-Thyroxine: results of a large controlled community-based questionnaire study. Clin Endocrinol (Oxf) 57:577-585

21. Gullo D, Latina A, Frasca F, Le Moli R, Pellgriti G, Vigneri R (2011) Levothyroxine Monotherapy cannot guarantee Euthyroidism in all Athyreotic patients. PLoSONE 6:e22552

22. Samuels MH, Kolobova I, Smeraglio A, Peters D, Purnell J, Schuff G (2016) Effects of Levothyroxine replacement therapy on energy expenditure and body composition. Thyroid 26:347-355

23. McAninch EA, Rajan KB, Miller $H$, Bianco AC (2018) Systemic thyroid Hormon status during Levothyroxine therapy in hypothyroidism: a systematic review and meta-analysis. J Clin Endocrinol Metab 103:4533-4542

24. Clyde PW, Harari AE, Getka EJ, Shakir KMM (2003) Combined Levothyroxine plus Liothyronine compared with Levothyroxine alone in primary hypothyroidism. A randomized controlled trial. JAMA 290:2952-2958

25. Jonklaas J, Tefera E, Shara N (2019) Prescribing therapy for hypothyroidism: influence of physician characteristics. Thyroid 29:44-52

26. Panicker V, Saravanan P, Vaidya B, Evans J, Hattersley AT, Frayling TM, Dayan CM (2009) Common variation in the $\mathrm{DIO} 2$ gene predicts baseline psychological well-being and respoonse to combination Thyroxine plus Triiodothyronine therapy in hypothyroid patients. J Clin Endocrinol Metab 94:1623-1629

27. Henessey JV (2015) Historical and current perspective in the use of thyroid extracts for the treatment of hypothyroidism. Endocr Pract 21:1161-1170

28. Hoang TD, Olsen CH, Mai VQ, Clyde PW, Shakir MKM (2013) Desiccated thyroid extract compared with Levothyroxine in the treatment of hypothyroidism: a randomized, double-blind, crossover study. JClin Endocrinol Metab 98:1982-1990

29. Tariq $A$, Wert $Y$, Cheriyath $P$, Joshi $R$ (2018) Effects of long-term combination LT4 und LT3 therapy for improving hypothyroidism and overall quality of life.Southern Med J 111:363-369

30. Shrestha A, Malabanan A, Haugen BR, Levy EG, Henessey JV (2017) Adverse event reporting in patients treated with thyroid hormone extract. Endocr Pract 23:566-575

31. Peterson SJ, Cappola AR, Castro MR, Dayan CM, Farwell AP, Henessey JV, Koop PA, Ross DS, Samuels MH, Sawka AM, Taylor PN, Jonklaas J, Bianco AC (2018) An online survey of hypothyroid patients demonstrates prominent dissatisfaction. Thyroid 28:707-721

Hinweis des Verlags. Der Verlag bleibt in Hinblick auf geografische Zuordnungen und Gebietsbezeich nungen in veröffentlichten Karten und Institutsadressen neutral.

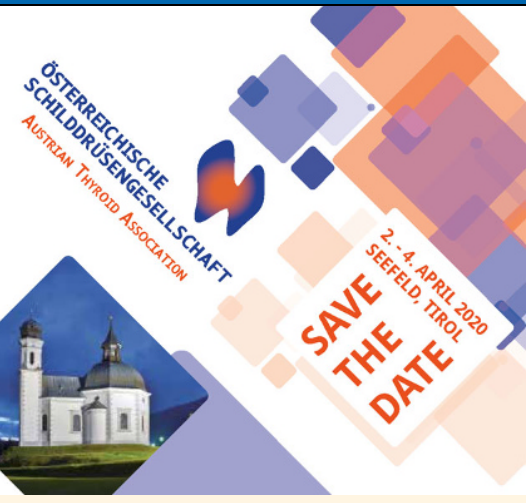

Schilddrüsen-Dialog 2020

Seefeld, Tirol - Olympia Sport- und Kongresszentrum, 2.-4. April 2020

\section{Themen}

- Thyreologie - Unterschiede USA - Europa

- Thyreologie und Gesundheitspolitik

- Schilddrüsenkarzinom

- Schilddrüsenerkrankungen beim Kind

- Blutungskomplikationen

- Das Leben ohne Schilddrüse

Schilddrüsenultraschall - Aufbaukurs am 2.4.2020 (begrenzte Teilnehmerzahl)

Im Mittelpunkt des Treffens soll der interdisziplinäre Austausch zwischen Nuklearmedizinern, Endokrinologen, Chirurgen, Pathologen, Gynäkologen, HNO-Ärzten sowie allen anderen thyreologisch interessierten Kolleginnen und Kollegen stehen.

\section{Kongressorganisation}

PCO Tyrol Congress

T: $+43(0) 512575600$

F: $+43(0) 512575607$

E: osdg@cmi.at

I: www.cmi.at

\section{Ausstellungsorganisation}

\section{\& Sponsoring}

S12! studio12 gmbh

T: +43 (0)512 890438

E: office@studio12.co.at

I: www.studio12.co.at

\section{Geschäftsstelle der OSDG}

Hermanngasse 18/1/4

A-1070 Wien

E: osdg@studio12.co.at

I: www.schilddruesengesellschaft.at

I: www.osdg.at 